\title{
Dispersion of solid saltating particles in a turbulent boundary layer
}

\author{
H. T. Wang ${ }^{1}$, Z. B. Dong ${ }^{1}$, X. H. Zhang ${ }^{2}$ \& M. Ayrault ${ }^{2}$ \\ ${ }^{1}$ Key Laboratory of Desert and Desertification, CAREERI, CAS, \\ Lanzhou Gansu, People's Republic of China \\ ${ }^{2}$ Laboratoire de Mécanique des Fluides et d'Acoustique, \\ UMR CNRS Ecully, France
}

\begin{abstract}
A horizontal saltation layer of glass particles in air is investigated experimentally both on a flat bed and over a two-dimensional ridge. Particle concentrations are measured by Mie scattering diffusion. All the statistical moments of the particle concentration are determined such as mean concentration, rms concentration fluctuations, skewness and flatness coefficients. Measurements of particle concentrations were made at the top of the ridge and in the cavity region. It is confirmed that over a flat bed the mean concentration decreases exponentially with height, the mean dispersion height $H$ being of great meaning. It is shown that the concentration distribution follows quite well a lognormal distribution. At the top of the ridge, the saltation layer is decreased and the concentration increased.
\end{abstract}

Keywords: particles, saltation, dispersion, turbulent boundary layer.

\section{Introduction}

Wind erosion is a major cause of soil degradation in arid and semi-arid areas and deserts. It is therefore of great importance to analyze both experimentally and theoretically the motion of wind-blown particles in order to develop effective wind erosion modeling system for controlling the wind erosion of small particles.

Saltation is the primary wind erosion mechanism. Initially aerodynamically lifted off by the wind in short hops and although accelerated by the wind, particles will return to the bed and will impact the ground, rebounding and/or 
ejecting other particles. The impact of saltating sand particles have a severe impact on the natural environment and human activity as for example soil erosion and dust entrainment (Shao et al. 1993).

In the present study, the dispersion of solid glass particles over a flat bed and over a two-dimensional triangular ridge is investigated experimentally by visualizing the wind-blown particles. Detailed measurements of concentration were made over a flat bed, at the hillcrest and in the near wake region. The saltation layer being considered as a continuum and using the Mie scattering diffusion properties, we present the particle concentrations statistics.

\section{Experimental set-up}

Different experiments were run in the blowing sand wind tunnel in the Key Laboratory of Desert and desertification, the Chinese Academy of Science, PR China (Dong et al. 2002). Particles used are glass particles with a density $\rho_{p}=2650 \mathrm{kgm}^{-3}$ and a mean diameter of $208 \mu \mathrm{m}$. The floor of the wind tunnel was covered with particles to a depth of $0.02 \mathrm{~m}$, installed $8 \mathrm{~m}$ downstream of the entrance of the test section and over a length of more than $3 \mathrm{~m}$. The measure area was set at $3 \mathrm{~m}$ from the upwind edge of the sand bed. The laser tomography visualizations were made in the vertical plane on the longitudinal axis of the wind tunnel.

The two-dimensional symmetrical triangular ridge was set at $3 \mathrm{~m}$ from the upwind edge of the sand bed (Figure 1). The height $h=24 \mathrm{~mm}$ (half the base width $B=48 \mathrm{~mm}$ ) is corresponding to the mean Eulerian dispersion height $H$ of the saltation layer or centroid on a flat bed (Zhang et al., 2004).

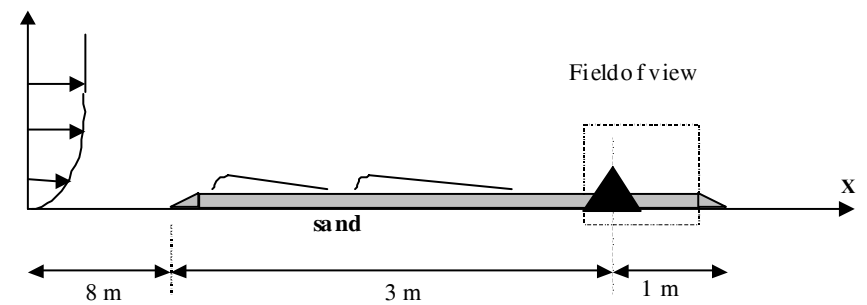

Figure 1: Sketch of the wind tunnel.

Two different experiments were run, over a flat bed for three different external velocities $\left(U_{e}=6 \mathrm{~ms}^{-1,} U_{e}=8 \mathrm{~ms}^{-1,}\right.$ and $\left.U_{e}=10 \mathrm{~ms}^{-1}\right)$ and over a steep twodimensional ridge for $U_{e}=8 \mathrm{~ms}^{-1}$. For the concentration field the saltation layer should be considered as a continuum, camera had a large field of view and the distance between two pixels was $\Delta p i x=0.186 \mathrm{~mm}$.

As discussed in detail by Ayrault and Simoens (1995) for the polydispersed incense particles, the grey level of the scattered light is proportional to $\sum d_{i}^{2} N_{i}$ 
where $N_{i}$ is the number density of particles of diameter $d_{i}$, and the subscript $i$ denotes the ensembles of different particle diameters in the total sample. This is true provided the absorption of light along the optical path, background illumination, geometrical distortions effects have been account for. Figure 2 represents the instantaneous image over a flat bed and the mean concentration of particles over the ridge image, the mean ensemble average being obtained from a set of 400 instantaneous images.
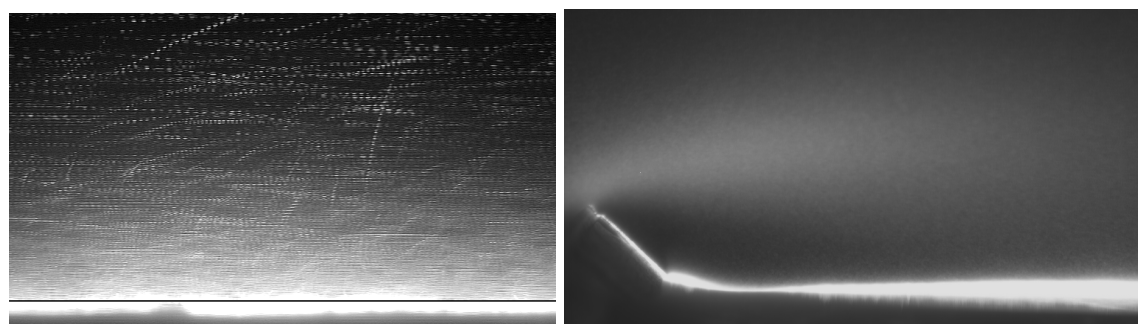

Figure 2: Example of (a) the instantaneous particle image and (b) the mean image concentration of particles over a flat bed and over a triangular ridge for $U_{e}=8 \mathrm{~ms}^{-1}$.

\section{Experimental results}

\subsection{Dispersion over a flat bed}

Figure 3 shows the vertical profiles of mean concentration expressed in grey level values for the three external velocity speeds $U_{e}=6,8,10 \mathrm{~ms}^{-1}$. The mean concentration profiles agree well with the exponential decay with height, which has been found previously by Nalpanis et al. (1993), Zhou et al. (2001) and Dong et al. (2002). Due to the intense reflection of light on the sand bed as clearly seen in figure 4, we couldn't determine the maximum of concentration at the sand level. Particularly for the $U_{e}=10 \mathrm{~ms}^{-1}$ speed of the free stream flow, the very high number of particles ejected causes some absorption of light along the optical paths near the sand bed, this absorption being not negligible. On account of the good agreement with the exponential fit, extrapolation was carried out by using the exponential fit curve and so, the maximum value was estimated and noted $<C(z=0)>$ estimated $=<C>\max$.

Profiles of the normalized mean particle concentration $\langle C(z)>/<C\rangle_{\max }$ against the normalized height $z / H$ are shown in figure 4 . The saltation layer or centroid. Is defined by

$$
H=\frac{\int_{0}^{\infty} z<C(x, z)>d z}{\int_{0}^{\infty}<C(x, z)>d z}
$$


When the height is normalized by the mean dispersion height $H$, all the data collapse together, profiles are similar and are in good agreement with the exponential form, except the near bed region for the $U_{e}=10 \mathrm{~ms}^{-1}$ fluid velocity speed. The mean dispersion height should be a characteristic length scale, the mean concentration could be expressed as

$$
\frac{<C(z)>}{<C(z=0)>}=\exp \left\{-\frac{z}{H}\right\}
$$

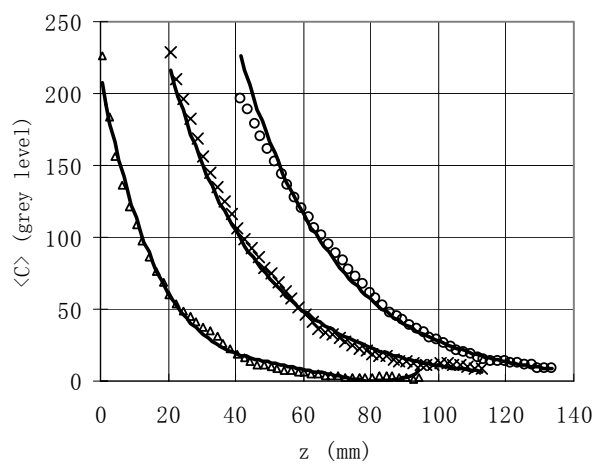

Figure 3: Mean concentration profiles (in grey level values) against vertical distance (in millimeters) for $U_{e}=6,8,10 \mathrm{~ms}^{-1}$. Profiles for 8 and $10 \mathrm{~ms}^{-1}$ have been translated: for $8 \mathrm{~ms}^{-1},(z+20)(\mathrm{mm})$; for $10 \mathrm{~ms}^{-1}$, $(z+40)(\mathrm{mm})$. Triangles: $U_{e}=6 \mathrm{~ms}^{-1}$; crosses: $U_{e}=8 \mathrm{~ms}^{-1}$; circles: $U_{e}=10 \mathrm{~ms}^{-1}$; Lines: best exponential fit.

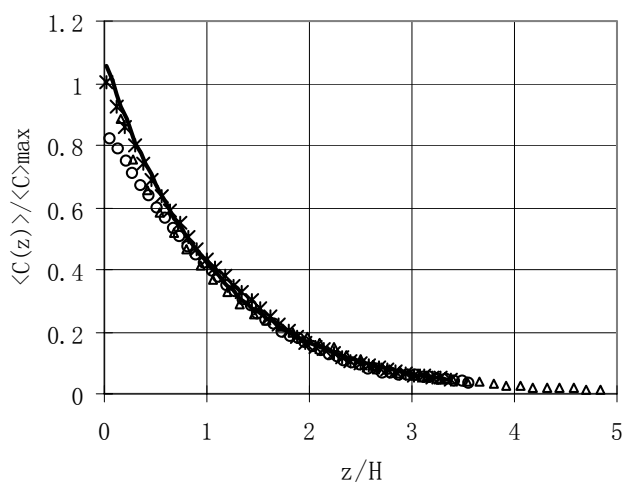

Figure 4: Non dimensionalized mean concentration profiles $<C(z)>/<C>\max$ against non dimensionalized height $z / H$ for the three $U_{e}=6,8,10 \mathrm{~ms}^{-1}$. Triangles: $U_{e}=6, \mathrm{~ms}^{-1}$; crosses: $U_{e}=8 \mathrm{~ms}^{-1}$; circles: $U_{e}=10 \mathrm{~ms}^{-1}$. Line: best exponential fit for $U_{e}=8 \mathrm{~ms}^{-1}$. 
The intensity of concentration $I(z)=\frac{c^{\prime}(z)}{\langle C(z)\rangle}$ is shown in figure 5. All the profiles exhibit the same shape but no clear similarity is found. As usual in such semi-bounded flows, the maximum intensity concentration values are obtained at the outer region of the saltation layer, where the mean concentration is small and the intermittency large.

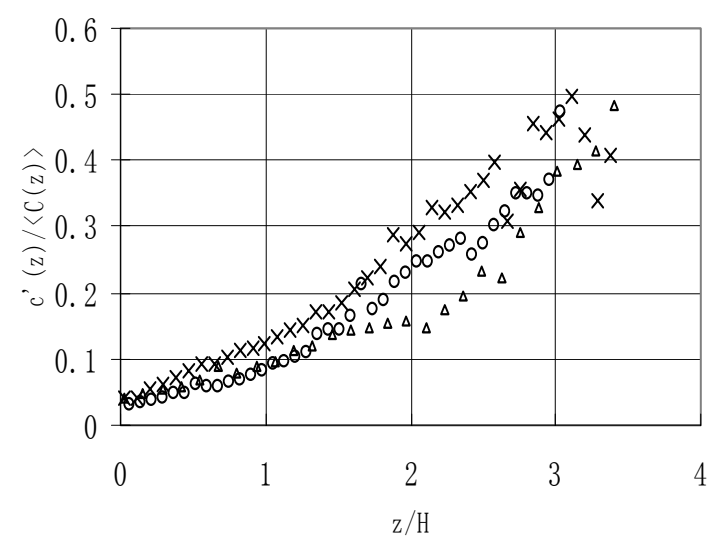

Figure 5: Intensity concentration profiles $I(z)=\frac{c^{\prime}(z)}{\langle C(z)\rangle}$ against $z / H$ for $U_{e}=6,8,10 \mathrm{~ms}^{-1}$. Triangles: $U_{e}=6 \mathrm{~ms}^{-1}$; crosses: $U_{e}=8 \mathrm{~ms}^{-1}$; circles: $U_{e}=10 \mathrm{~ms}^{-1}$.

The experimental probability density functions at different heights $z=H / 2, H, 2 H$ inside the saltation layer are plotted in figure 6 for $U_{e}=8 \mathrm{~ms}^{-1}$. The agreement of the experimental values with the theoretical lognormal distribution is quite reasonable. So we could consider that inside the saltation layer and for the three velocity speeds studied, the concentration of saltating sand particles are lognormal distributed.
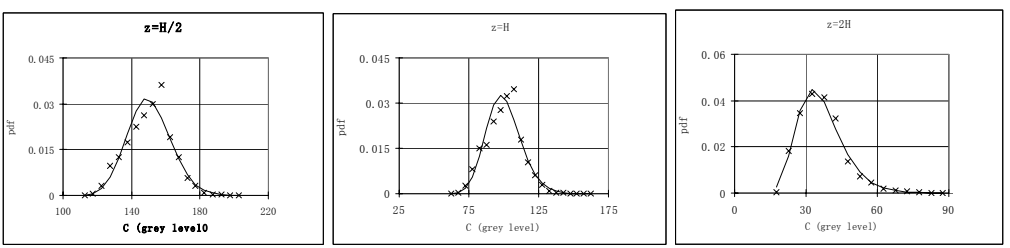

Figure 6: Pdf of concentration at $z=H / 2, H, 2 H$ for $U_{e}=8 \mathrm{~ms}^{-1}$. Experimental results: symbols; theoretical curve: line. 


\subsection{Dispersion over a two dimensional ridge}

The influence of the ridge on the particle concentration was also investigated. Although the fluid flow is accelerated over the ridge, particles are decelerated due to the effects of the gravity. The mean concentration profiles were measured at six positions along the center line of the wind tunnel: the hill top, the downwind hill foot $\mathrm{X}=1 h, \mathrm{X}=2 h, \mathrm{X}=4 h, \mathrm{X}=6 h$ and $\mathrm{X}=12 h$. Figure 7 shows the different vertical profiles of mean concentration $\langle C(z)>$ normalized by the maximum value of the mean concentration at the hill top $\left\langle C_{X=0}>_{\max }\right.$. As clearly seen, all these profiles exhibit very different shapes. The theoretical exponential law is quite well verified at the top. As expected, the other profiles in the near wake have different shapes with elevated maxima as profiles for mean and rms concentrations.

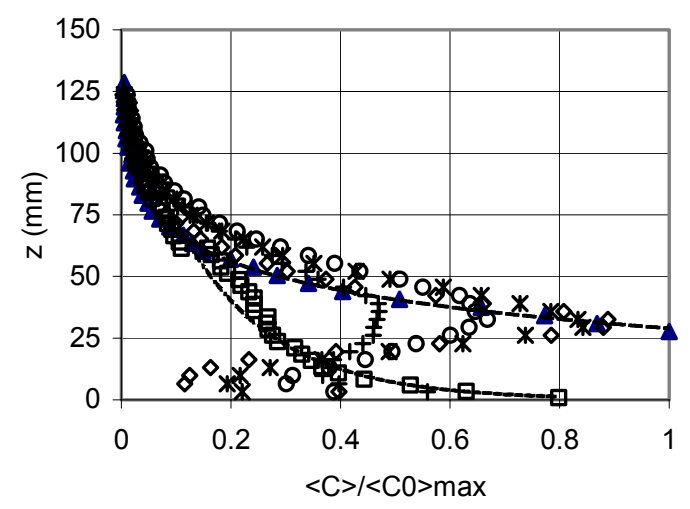

Figure 7: Vertical mean concentration profiles at six downwind positions from the ridge top. Triangles $X=0 \mathrm{~h}$; inclined squares: $X=1 \mathrm{~h}$; crosses: $X=2 \mathrm{~h}$; circles: $X=4 \mathrm{~h}$; plus: $X=6 \mathrm{~h}$; squares: $X=12 \mathrm{~h}$.

The fit line for $\mathrm{X}=0$ represents the theoretical curve $\frac{\langle C(z)\rangle}{\langle C(z=0)\rangle}=\exp \left(-\frac{z}{H}\right)$.

The vertical profiles of the rms of particle concentration exhibit elevated maxima, except for the last section in the wake. The heights of the maxima increase with the hill distance with a relatively high value, more than $15 \%$. The vertical profiles of the intensity of concentration $I(z)=\frac{c^{\prime}(z)}{\langle C(z)\rangle}$ shown in figure 8 exhibit the same shape but no clear similarity is found. As usual in such semibounded flows, the maximum intensity concentration values are obtained in the outer region of the saltation layer, where the mean concentration is small and the intermittency large. 


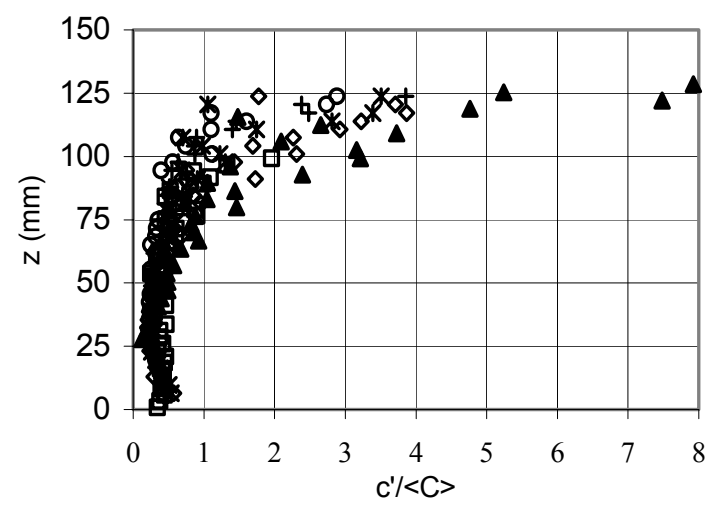

Figure 8: Vertical rms profiles at six downwind positions from the ridge top. Triangles $X=0 \mathrm{~h}$; inclined squares: $X=1 \mathrm{~h}$; crosses: $X=2 \mathrm{~h}$; circles: $X=4 \mathrm{~h}$ plus: $X=6 \mathrm{~h}$; squares: $X=12 \mathrm{~h}$.

From the skewness and flatness profiles, it was shown that the concentration distribution is not following a lognormal distribution (Figure 9).

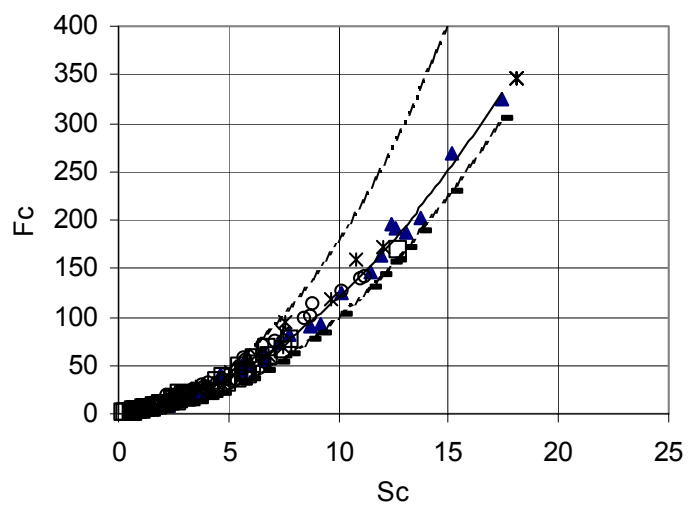

Figure 9: Flatness versus Skewness profiles at four downwind positions downwind the ridge. Triangles $X=0 h$; crosses: $X=2 h$; circles: $X=4 h$; squares: $X=12 h$. Dashed line: $K=S_{2}+1$; dashed line: $K=\frac{16}{9} S^{2}+3$; line: parabola for $X=4 h$.

Hill induced perturbations in the mean concentration were determined from their measured profiles with and without the ridge. The vertical profiles of 
fractional concentration-up ratio $\Delta C$, defined by $\Delta C=\frac{\langle C(z)\rangle}{\langle C\rangle_{\max }}-\frac{\left\langle C_{f}(z)\right\rangle}{\left\langle C_{f}\right\rangle_{\max }}$ where $\mathrm{z}$ is the height above the lower surface and $\left\langle C_{f}(z)\right\rangle$ is the corresponding mean concentration above the flat surface are shown in figure 10. A reduction in concentration with a minimum of -0.25 and -0.5 close to the surface, is observed respectively at the two sections $X=2 h$ and $X=4 h$. A positive perturbation persists at heights greater than respectively $10 \mathrm{~mm}$ and $15 \mathrm{~mm}$ above the sand bed. We note that no negative value is observed for the $\mathrm{X}=6 \mathrm{~h}$ section. In the last section, $\mathrm{X}=12 \mathrm{~h}$, only small positive fractional concentration-up values of about 0.2 exist. The particle flow is nearly recovering his behavior without a ridge.

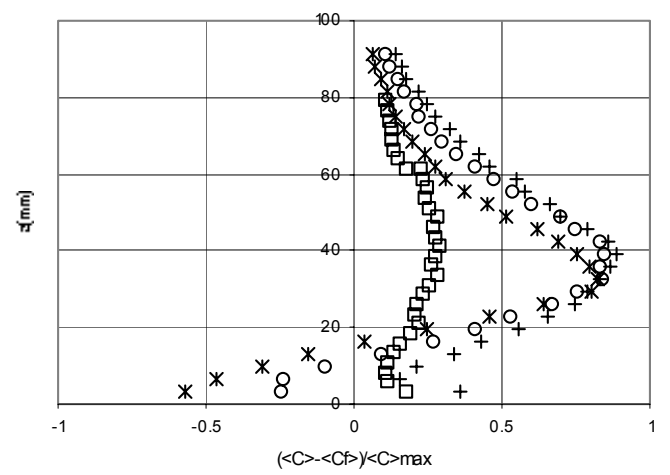

Figure 10: Fractional concentration-up factor at four downwind positions. Crosses: $X=2 h$; circles: $X=4 h$; plus: $X=6 h$; squares: $X=12 h$.

\section{Conclusion}

A horizontal saltation layer of glass particles in air was investigated experimentally. Particle concentrations were measured by Mie scattering diffusion (MSD). All the statistical moments of the particle concentration were determined such as mean and rms concentration, skewness and flatness coefficients, probability density functions.

It was found that the mean concentration over a flat bed is decreasing exponentially with height, the mean dispersion height $H$ being of great meaning. In this framework, the saltation layer could be investigated as composed of two adjacent layers. The inner saltation layer where particles are mainly influenced by the bed effects and the outer saltation layer where this is the external fluid flow which greatly influences particles. It was also shown that the concentration distribution is following quite well a lognormal distribution.

The influence of the ridge on the particle concentration was also investigated. At the hill top, the saltation layer height is smaller than in flat terrain. The 
theoretical exponential law is quite well verified at the top and also, for $\mathrm{X} / \mathrm{h}=12$, the vertical profile is near this curve. Although the fluid flow is accelerated over the ridge, particles are decelerated due to the effects of the gravity with a minimum speed-up factor of about 0,4 at the hill top. On the lee side, there is a deceleration of the particles and an increase of the velocity fluctuations. As expected, the other profiles have different shapes with elevated maxima as profiles for mean and rms concentrations. From the skewness and flatness profiles, it was shown that the concentration distribution is not following a lognormal distribution.

\section{References}

[1] Ayrault M. and Simoens S. 1995, J. Flow Visualization Image Processing 2, 195-208.

[2] Dong Z., Liu X., Li F., Wang H. and Zhao A. 2002, Earth Surface Processes and Landforms 27, 641-658.

[3] Nalpanis, P., Hunt, J.C.R., Barret, C.F., 1993, Saltating particles over flat beds, J. Fluid Mech., vol 251, 661-685.

[4] Dong Z., Wang H., Liu X. Li F. And Zhao A., 2002, Geomorphology 45, 277-289.

[5] Shao Y., Raupach M.R. and Findlater P.A. 1993, J. Geophysical Res., vol. 98, D7, 12719-12726.

[6] Zou, X.Y., Wang, Z.L., Hao, Q.Z., Zhang, C.L., Liu, Y.Z., Dong, G.R., 2001, The distribution of velocity and energy of saltating grains in a wind tunnel, Geomorphology 36, 155-165. 